University of Montana

ScholarWorks at University of Montana

$1-1997$

\title{
Recovery of Crenarchaeotal Ribosomal DNA Sequences from Freshwater-Lake Sediments
}

Christa Schleper

William E. Holben

University of Montana - Missoula, Bill.Holben@mso.umt.edu

Hans-Peter Klenk

Follow this and additional works at: https://scholarworks.umt.edu/biosci_pubs

Part of the Biology Commons

Let us know how access to this document benefits you.

\section{Recommended Citation}

Schleper, Christa; Holben, William E.; and Klenk, Hans-Peter, "Recovery of Crenarchaeotal Ribosomal DNA Sequences from Freshwater-Lake Sediments" (1997). Biological Sciences Faculty Publications. 111. https://scholarworks.umt.edu/biosci_pubs/111

This Article is brought to you for free and open access by the Biological Sciences at ScholarWorks at University of Montana. It has been accepted for inclusion in Biological Sciences Faculty Publications by an authorized administrator of ScholarWorks at University of Montana. For more information, please contact scholarworks@mso.umt.edu. 


\title{
Recovery of Crenarchaeotal Ribosomal DNA Sequences from Freshwater-Lake Sediments
}

\author{
CHRISTA SCHLEPER, ${ }^{1 *}$ WILLIAM HOLBEN, ${ }^{2}$ AND HANS-PETER KLENK ${ }^{3}$ \\ Marine Science Institute, University of California, Santa Barbara, California $93106^{1}$; Division of \\ Biological Science, University of Montana, Missoula, Montana 59812²; and \\ The Institute for Genomic Research, Rockville, Maryland $20850^{3}$
}

Received 12 July 1996/Accepted 22 October 1996

\begin{abstract}
We report several novel environmental sequences of archaea from the kingdom Crenarchaeota, recovered from anaerobic freshwater-lake sediments in Michigan. A nested PCR approach with Archaea- and Crenarchaeota-specific primers was used to amplify partial Small-subunit ribosomal DNAs. Phylogenetic analysis of seven sequences shows that these DNAs represent a monophyletic lineage diverging prior to all recently identified crenarchaeotal phylotypes isolated from temperate environments. Including our lineage, all uncultured crenarchaeotal sequences recovered from moderate or cold environments form a distinct, monophyletic group separate from the "genuine" thermophilic crenarchaeota. Our finding extends the emerging picture that crenarchaeota, thought until recently to be solely extreme thermophiles, have radiated into an unexpectedly large variety of ecologically important, temperate environments.
\end{abstract}

Recent molecular phylogenetic studies based on $16 \mathrm{~S}$ ribosomal DNA (rDNA) analysis of microbial assemblages indicate a much greater phylogenetic and probably also physiological diversity of Archaea than previously assumed. In particular, two major discoveries in this regard have been made for the kingdom Crenarchaeota, a group originally thought to consist solely of extremely thermophilic organisms with low evolutionary rates. First, many novel phylotypes of phenotypically undescribed crenarchaeota have been isolated from a hot spring in Yellowstone National Park, indicating an unexpectedly high phylogenetic diversity in this habitat $(1,2)$. Second, crenarchaeotal phylotypes have also been detected in nonthermophilic marine environments, i.e., in temperate subsurface ocean waters $(3,6)$ and polar seas (4) and in association with marine invertebrates $(10,13)$. More recently, a short 16S rDNA fragment with crenarchaeotal affiliation has also been amplified from the soil microbial community of a soybean field in Japan (16).

Actively dividing cells of a crenarchaeote living in the tissues of a temperate marine sponge have been visualized by wholecell hybridization analyses, demonstrating growth of this organism in temperatures as low as $10^{\circ} \mathrm{C}$ (13). Furthermore, crenarchaeotal rRNA was found to represent a significant fraction of the prokaryotic biomass in coastal Antarctic surface waters (4). These data are good evidence for the occurrence of crenarchaeota of as-yet-undescribed phenotypes in temperate or cold environments and suggest that these crenarchaeota are ecologically important for the oceans.

In order to identify nonthermophilic crenarchaeota in other habitats, we searched for phylogenetic lineages of this group in the sediments of two freshwater lakes by using a $16 \mathrm{~S}$ rDNAbased PCR approach. Samples were taken from different depths ( 0 to 2,4 to 6 , and 6 to $8 \mathrm{~cm}$, respectively) of the sediments from the eutrophic Lake Wintergreen (samples designated Win) and the oligotrophic Lawrence Lake (samples designated Law), both located in Michigan, near Kalamazoo.

\footnotetext{
* Corresponding author. Mailing address: Marine Science Institute, University of California, Santa Barbara, CA 93106. Phone: (805) 8938087. Fax: (805) 893-8062. E-mail: christa@anarchae.lscf.ucsb.edu.
}

Total bacterial community DNA was prepared by direct lysis as described previously (7). The procedure results in essentially quantitative recovery of bacterial DNA as determined by microscopic examination of samples after lysis and comparison to estimated yields (7). Fifty nanograms of each of these DNA samples was used as a template in an initial PCR amplification, with 16S rDNA primers specific for Archaea (Arch21F and Arch958R [3]). Reaction mixtures of $50 \mu$ l containing 125 pmol of each primer were used for thermal cycling (denaturation for $1 \mathrm{~min}$ at $95^{\circ} \mathrm{C}$, annealing for $1 \mathrm{~min}$ at $55^{\circ} \mathrm{C}$, and extension for $2 \mathrm{~min}$ at $72^{\circ} \mathrm{C}$; total of 30 cycles). Specific PCR products of the expected size $(950 \mathrm{bp})$ were obtained from three of the six samples, i.e., Win0-2, Law0-2, and Law4-6. As controls, primers specific for eubacteria (3) were used on all DNA samples. The primers yielded amplification products in all cases, indicating that the samples did not contain PCR inhibitors. Since methanogenic archaea are frequently found in anaerobic freshwater-lake sediments, we expected a large fraction of the archaeal PCR products to be of euryarchaeotal origin. In order to specifically amplify crenarchaeotal rDNA from these fragments, we designed a second primer set based on the $16 \mathrm{~S}$ rDNA alignment of known archaea, Cren28F (5'AAT CCG GTT GAT CCT GCC GGA CC-3') and Cren457R (5'-TTG CCC CCC GCT TAT TCS CCC G-3'). Due to mismatch pairing of the $3^{\prime}$ ends of these primers with rDNA of euryarchaeotal (and eubacterial or eukaryotic) origin, only products from crenarchaeota should be amplified with $3^{\prime}$ to $5^{\prime}$ exonuclease-free polymerase in the PCR mixture. The specificity of these primers was confirmed by reactions with chromosomal DNA of five euryarchaeotal and crenarchaeotal organisms. At an annealing temperature of $50^{\circ} \mathrm{C}$, PCR fragments of the expected size (470 bp) were obtained from all crenarchaeotal DNAs tested, i.e., from Sulfolobus solfataricus, Desulfurococcus mobilis, Thermoproteus tenax, Pycrobaculum aerophilum, and Pyrodictium occultum. Euryarchaeotal DNAs yielded negative results in all cases, as expected (i.e., Pyrococcus furiosus, Thermococcus celer, Methanothermus fervidus, Methanopyrus kandleri, and Methanococcus jannaschii). This primer set was used under the same conditions in a nested PCR on $3 \mu \mathrm{l}$ of each of the reaction products obtained from the lake sediments with the primers biased towards the archaea (Win0-2, Law0-2, 


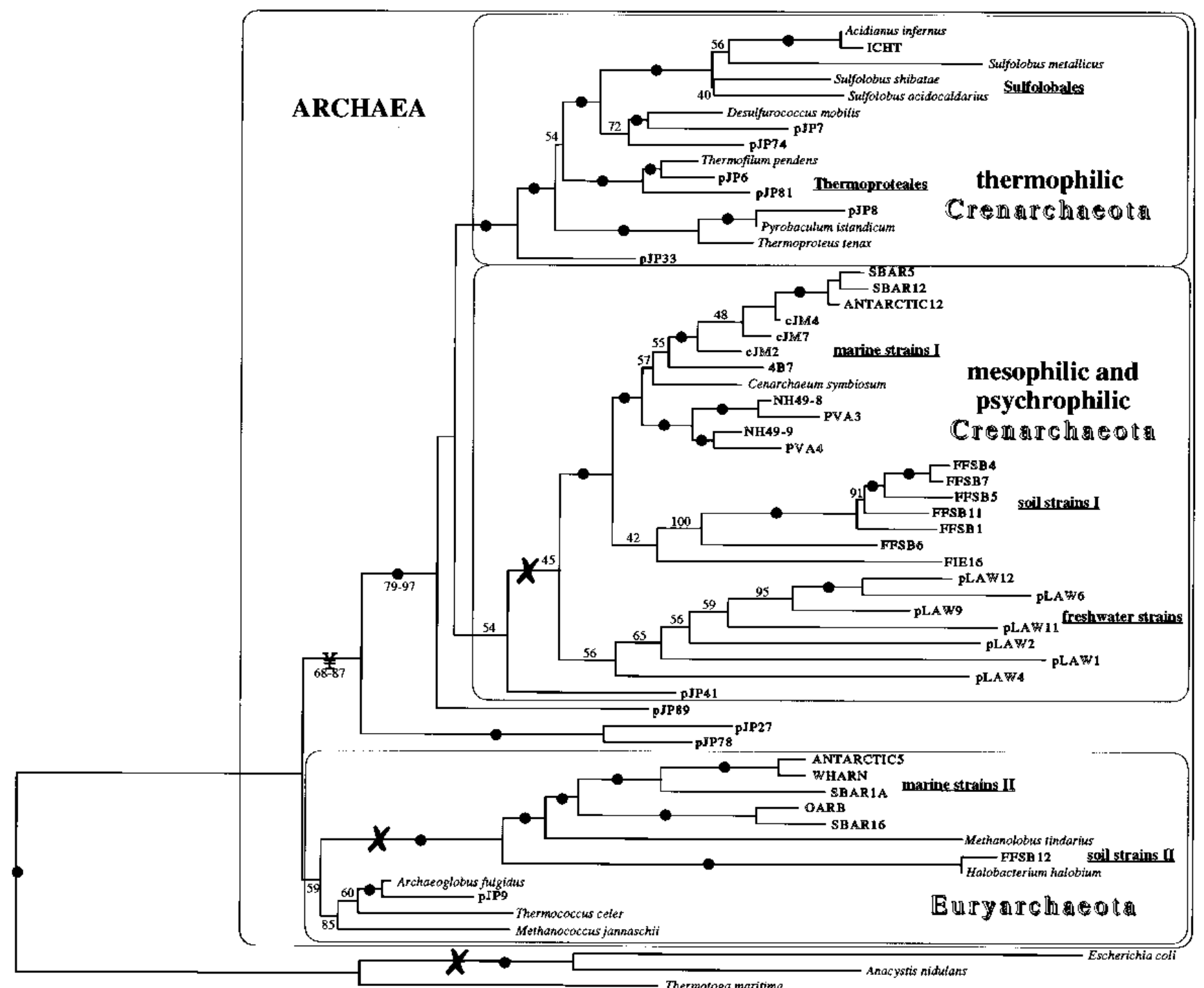

BACTERIA outgroup

FIG. 1. Phylogenetic tree of the Archaea, inferred by the neighbor-joining method (14) from sequences corrected under the Kimura two-parameter model (9). The cJM, PVA, and FIE branches were added to the tree, with lengths inferred by a complementary reconstruction based on a smaller data set, because these partial sequences have no overlaps with the new pLAW sequences. The phylogenetic analyses were performed with programs from the PHYLIP package (5). All sequences except the new pLAW sequences were accessed from public databases, GenBank (United States) and DDBJ (Japan). Abbreviations indicate uncultured phylotypes from various environments: ICHT, close relative of Acidianus (12); pJP, from a Yellowstone National Park hot spring (1); WHARN, OARB, and SBAR, from coastal marine environments (3); ANTARCTIC, from antarctic marine picoplankton (4); cJM, from a deep-sea deposit feeder (10); NH49, from marine picoplankton (6); PVA, from deep-water samples off Hawaii (11); FFSB, from the humus layer of Finnish forest soil (8); FIE16, from a Japanese soybean field (16); pLAW, from freshwater sediments (this study). Dots indicate 33 topological elements which were confirmed by all five bootstrap analyses performed: (i) neighbor-joining analysis based on identities only, (ii) neighbor-joining analysis based on transversions only, (iii) parsimony analysis based on identities only, (iv) parsimony analysis based on transversions only, and (v) neighbor-joining analysis inferred from distances corrected under the Kimura two-parameter model. The highest level of statistical support (bootstrap values, expressed as percentages) for the topological elements which are not shared by all five bootstrap analyses is indicated above the relevant branches. X, point of transition from hot to cold or moderate environments.

and Law4-6). Fragments of the expected size were amplified only from sample Law4-6, i.e., from an anaerobic fraction of the sediments of Lawrence Lake in which sulfide and methane were present. The temperature of the sediments was about $8^{\circ} \mathrm{C}$ at the time of sampling but presumably would be lower during the winter at this temperate latitude. The PCR fragments were purified (Qiaex; Qiagen) and were cloned with a commercially prepared vector (pGEM; Promega). Seven of the insert-containing clones from this library were subsequently subjected to a sequence analysis. The sequences were compared to all corresponding segments of $16 \mathrm{~S}$ rRNA genes from Crenarchaeota that are available in the databases, as well as to some representative euryarchaeotal and bacterial sequences, by the neighbor-joining method (14) for sequence distances corrected under the Kimura two-parameter model (9). The alignment included 20 sequences of cultivated thermophilic crenarchaeota as well as 33 sequences of as-yet-uncultivated phylotypes from this kingdom.

This analysis revealed that all of the sequences obtained from Lawrence Lake were related to each other and were clearly affiliated with Crenarchaeota. The $\mathrm{G}+\mathrm{C}$ content of the pLAW sequences was between $52.5 \%$ (pLAW1) and $58.6 \%$ 
(pLAW11), i.e., about $10 \%$ lower than in the respective regions in the rDNA genes of cultured crenarchaeotes but similar to the average $\mathrm{G}+\mathrm{C}$ content found in the sequences from the marine crenarchaeal plankton (52.4\% [3]). Secondary structure analysis did not identify any potentially artifactual chimeric molecules tha might have been created in the PCR.

Several phylogenetic analyses based on different assumptions for the reconstruction yielded essentially the overall tree topology shown in Fig. 1, with only minor variations in some elements (for details about the phylogenetic procedures, see the figure legend). Our phylogenetic analyses show that the Crenarchaeota are divided into two major clades. One of them contains all known cultivated thermophilic members of this kingdom as well as phylotypes obtained from a hot spring in Yellowstone National Park. The other clade includes all sequences of as-yet-uncultivated organisms recovered from moderate or cold environments and one phylotype from a hot spring (pJP41). Within this group, the lineage containing our sequences from Lawrence Lake forms the lowest branch of all nonthermophilic phylotypes. The unity of this lineage, however, received only 56\% support from bootstrapping analysis. With $97 \%$ bootstrap support, all other nonthermophilic crenarchaeota from marine and soil environments share an ancestor to the exclusion of the Lawrence Lake lineage. The only phylotype within this crenarchaeotal clade that has been isolated from a hot environment, pJP41, forms the lowest branch in the tree shown and is one of the most ambiguous lineages in the phylogeny. In three of the five analyses, pJP41 is found together with the "genuine" thermophiles, indicating that there may not be any phylotypes isolated from hot environments in this clade of the Crenarchaeota. Two environmental sequences, pJP27 and pJP78, that have recently been isolated from a hot spring in Yellowstone National Park (2) branch deeply within the Crenarchaeota, below the two major clades in our analysis. Barns et al. have suggested recognizing this lineage provisionally as a third archaeal kingdom, the "korarchaeota" (2), because they have found these sequences branching either deeply within the Crenarchaeota as well, below the bifurcation of Crenarchaeota and Euryarchaeota, or even as a sister group of eukaryotes, depending on the analytical method used. Our data suggest that there has been only one transition from thermophilic to mesophilic and psychrophilic phenotypes within the Crenarchaeota. However, this hypothesis may not stand the test of time, considering the rapidly growing number of novel phylotypes detected in diverse environments.

Our finding of crenarchaeotal phylotypes in nonthermophilic anaerobic sediments of an oligotrophic freshwater lake extends the emerging picture showing that members of this kingdom of the Archaea inhabit a wide variety of niches and are ecologically much more successful and important than was previously thought. The extent of phylogenetic diversity within the clade of nonthermophilic crenarchaeota and their occur- rence in many different environments strongly suggests that a variety of new and perhaps unique physiological phenotypes will be discovered as some of these organisms are eventually isolated in laboratory cultures and characterized.

Nucleotide sequence accession numbers. The sequences reported herein have been deposited in the GenBank database under accession numbers U77568 through U77575.

We are grateful to Melvin Simon and Edward DeLong for their generous support. We also thank Steve Marsh for technical assistance and Michael Klug of the Kellogg Biological Station for providing the sediment samples.

C.S. has a postdoctoral fellowship from the Deutsche Forschungsgemeinschaft.

\section{REFERENCES}

1. Barns, S. M., R. E. Fundyga, M. W. Jeffries, and N. R. Pace. 1994. Remarkable archaeal diversity detected in a Yellowstone National Park hot spring environment. Proc. Natl. Acad. Sci. USA 91:1609-1613.

2. Barns, S. M., C. F. Delwiche, J. D. Palmer, and N. R. Pace. 1996. Perspectives on archaeal diversity, thermophily and monophyly from environmental rRNA sequences. Proc. Natl. Acad. Sci. USA 93:9188-9193.

3. DeLong, E. F. 1992. Archaea in coastal marine environments. Proc. Natl. Acad. Sci. USA 89:5685-5689.

4. DeLong, E. F., K. Y. Wu, B. B. Prezelin, and R. V. M. Jovine. 1994. High abundance of Archaea in Antarctic marine picoplankton. Nature 371:695697.

5. Felsenstein, J. 1992. PHYLIP users manual V3.5. University of Washington, Seattle.

6. Fuhrman, J. A., K. McCallum, and A. A. Davis. 1993. Novel major archaebacterial group from marine plankton. Nature 356:148-149.

7. Holben, W. E. 1994. Isolation and purification of bacterial DNA from soil, p. 727-751. In R. W. Weaver et al. (ed.), Methods of soil analysis, part 2. Microbiological and biochemical properties. SSSA book series no. 5. Soil Science Society of America, Madison, Wis.

8. Jurgens, G. N. 1996. Archaeal sequences isolated from humus layer of Finnish forest soil. GenBank accession numbers X96688, X96691, X96692, X96693, X96694, and X96696.

9. Kimura, M. 1980. A simple method for estimating evolutionary rates of base substitutions through comparative studies of nucleotide sequences. J. Mol. Evol. 16:111-120.

10. McInerney, J. O., M. Wilkinson, J. W. Patching, T. M. Embley, and R. Powell. 1995. Recovery and phylogenetic analysis of novel archaeal rRNA sequences from a deep-sea deposit feeder. Appl. Environ. Microbiol. 61: $1646-1648$

11. Moyer, C. L., F. C. Dobbs, and D. M. Karl. GenBank accession numbers U46679 and U46680.

12. Norris, P. R., S. Waterhouse, and N. P. Burton. Unidentified archaeon ICHT 16S ribosomal RNA, complete sequence. GenBank accession number U38362.

13. Preston, C. M., K. Y. Wu, T. F. Molinski, and E. F. DeLong. 1996. A psychrophilic Crenarchaeote inhabits a marine sponge: Cenarchaeum symbiosum gen.nov., sp.nov. Proc. Natl. Acad. Sci. USA 93:6241-6246.

14. Saitou, N., and M. Nei. 1987. The neighbor-joining method: a new method for reconstructing phylogenetic trees. Mol. Biol. Evol. 4:406-425.

15. Stein, J. L., T. L. Marsh, K. Y. Wu, H. Shizuya, and E. F. DeLong. 1996. Characterization of uncultivated prokaryotes: isolation and analysis of a 40-kilobase-pair genome fragment from a planktonic marine archaeon. J. Bacteriol. 178:591-599.

16. Ueda, T., Y. Suga, and T. Matsuguchi. 1995. Molecular phylogenetic analysis of a soil microbial community in a soybean field. Eur. J. Soil Sci. 46:415-421. 